\title{
Localized solid-state amorphization at grain boundaries in a nanocrystalline Al solid solution subjected to surface mechanical attrition
}

\author{
$\mathbf{X} \mathbf{W u}^{1,2}, \mathbf{N} \mathbf{T a o}^{2}, \mathbf{Y} \mathrm{Hong}^{1}, \mathbf{J} \mathbf{L u}^{3,4}$ and $\mathbf{K} \mathbf{L u}^{2}$ \\ ${ }^{1}$ State Key Laboratory of Nonlinear Mechanics, Institute of Mechanics, Chinese Academy of \\ Sciences, Beijing 100080, People's Republic of China \\ ${ }^{2}$ Shenyang National Laboratory for Materials Science, Institute of Metal Research, Chinese \\ Academy of Sciences, Shenyang 110016, People's Republic of China \\ ${ }^{3}$ LASMIS, University of Technology of Troyes, 10000, Troyes, France \\ E-mail: xlwu@imech.ac.cn
}

Received 10 June 2005, in final form 2 September 2005

Published 7 November 2005

Online at stacks.iop.org/JPhysD/38/4140

\begin{abstract}
Using high-resolution electron microscopy, localized solid-state amorphization (SSA) was observed in a nanocrystalline (NC) Al solid solution (weight per cent $4.2 \mathrm{Cu}, 0.3 \mathrm{Mn}$, the rest being $\mathrm{Al}$ ) subjected to a surface mechanical attrition treatment. It was found that the deformation-induced SSA may occur at the grain boundary (GB) where either the high density dislocations or dislocation complexes are present. It is suggested that lattice instability due to elastic distortion within the dislocation core region plays a significant role in the initiation of the localized SSA at defective sites. Meanwhile, the GB of severely deformed $\mathrm{NC}$ grains exhibits a continuously varying atomic structure in such a way that while most of the GB is ordered but reveals corrugated configurations, localized amorphization may occur along the same GB.
\end{abstract}

\section{Introduction}

Solid-state amorphization (SSA) via mechanical deformation has been the focus of many theoretical and experimental investigations in recent years [1-8]. SSA is ubiquitous. It occurs in metallic alloys, ceramics and some covalently bonded pure elements such as $\mathrm{Si}$ and can be achieved through various mechanical deformation methods often with extreme conditions of severe strains and/or high strain rates. Up to now, the underlying microscopic mechanisms of SSA have not been unambiguously recognized. However, of particular significance is the finding that the amorphous phase commonly nucleates at the various defect sites, e.g. microband [9], twin and lattice dislocations $[10,11]$ and grain boundary (GB) [11]. It turns out to be a general rule that either the high defect concentration or dislocation activity plays a crucial role in SSA.

\footnotetext{
4 Present address: Department of Mechanical Engineering, Hong Kong Polytechnic University, Hung Hom, Kowloon, Hong Kong, People's Republic of China.
}

On the other hand, there are several theoretical models but they lack experimental backing. For example, the nucleation of the amorphous phase is considered to occur at the cores of the lattice and interfacial dislocations and disclinations via a process associated with the special splitting of such defects $[6,12]$. Theoretical modelling [7], using molecular dynamics (MD) simulations in binary substitutional solid solutions, demonstrated that dislocations are responsible for crystalline phase instability and that the nature of SSA is determined by defect properties such as formation energy and density, and by kinetic constraints. The MD simulation [5] of single crystal Ni and $\mathrm{NiCu}$ nanowires deformed to $100 \%$ strain at high rates of $5 \% \mathrm{ps}^{-1}$ exhibits a continuous process of amorphizing a solid with a dramatic change in atomic short-range order and loss of shear rigidity. The amorphization may occur directly from the homogeneous, elastically deformed system with no chemical or structural inhomogeneities. Very recently it was reported that the shock-induced SSA phenomenon in boron carbide is ascribed to the large elastic strains during shock loading and 


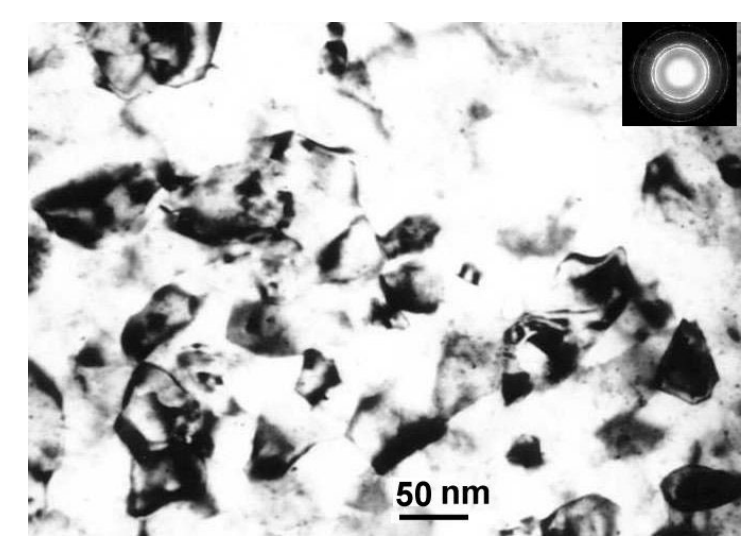

Figure 1. Electron micrograph of NC grains. Inset is the corresponding SAED pattern.

the subsequent violation of the Born stability conditions, which lead to crystal collapse [13].

In this paper, high-resolution electron microscopy (HREM) observations have been performed to inspect the micromechanism of SSA in a severely deformed Al solid solution subjected to severe plastic deformation.

\section{Experimental}

The material used in this investigation was an $\mathrm{Al}$ alloy with the chemical composition (in wt $\%$ ) of $4.2 \mathrm{Cu}, 0.3 \mathrm{Mn}$, the rest being Al. The samples were $100 \mathrm{~mm}$ in diameter and $10 \mathrm{~mm}$ in thickness. A solution heat treatment was conducted to obtain a single solid solution phase with homogeneous grains of about $30 \mu \mathrm{m}$.

The surface mechanical attrition treatment (SMAT) was used to impose large strains together with high strain rates on the surface layer of the materials. The SMAT technique was described in detail in our previous papers [14-17]. In brief, during the SMAT process, the hardened steel balls $8 \mathrm{~mm}$ in diameter were placed at the bottom of a cylindershaped vacuum chamber attached to a vibration generator, with which the balls were resonated. Because of the high vibration frequency of the system, the sample surface was peened repetitively by a large number of balls within a short period of time. Hence, the grains in the treated layer were effectively refined to nanoscale [14]. In the present work, the SMAT process was performed for $50 \mathrm{~min}$ at room temperature with a vibrating frequency of $50 \mathrm{~Hz}$ in a vacuum.

Following the SMAT, the microstructure characterization was performed in a HREM (JEM2010F) operated at $200 \mathrm{kV}$. Both cross-sectional and plane-view thin foils were prepared for HREM observations [14, 15].

\section{Results}

The nanocrystalline (NC) grains $(<100 \mathrm{~nm}$ in size $)$ were present from $\sim 27 \mu \mathrm{m}$ deep to the top surface of the treated layer. Figure 1 shows a typical microstructure with the corresponding selected area electron diffraction (SAED) pattern. The pattern exhibits rings, indicating high angle GBs and random crystallographic orientations in the selected field of view. Some GBs are poorly defined and the contrast
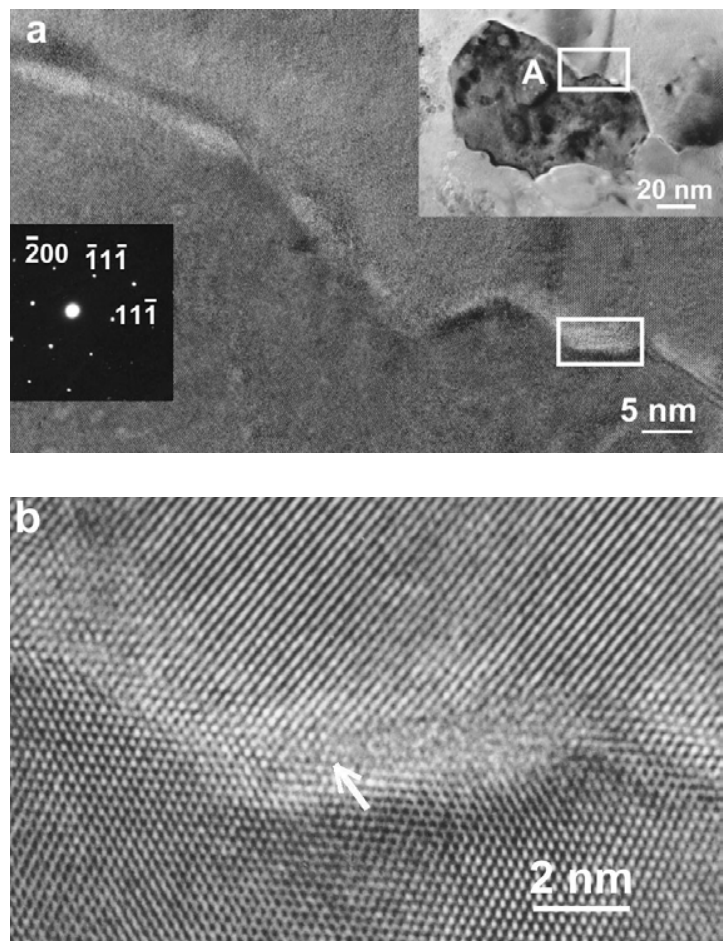

Figure 2. (a) Electron micrograph of the local GB with the corrugated configuration. Top-right inset shows the NC grain. Bottom-left inset is the SAED pattern with [011] zone axis. (b) HREM image of the GB of the rectangle part of $(a)$, showing an amorphous structure along the GB. The arrow indicates the severe lattice distortion in the vicinity of the amorphous phase.

within the grain is not uniform, but often changes in a complex fashion that indicates a high level of internal stresses and elastic distortions in the crystal lattice due to the presence of a high density of dislocations at the boundaries. X-ray diffraction analysis indicates that no other phases, but the fcc Al phase are detected in the treated layer.

Figure 2(a) shows an NC grain of $\sim 120 \mathrm{~nm}$ in diameter (top-right inset), its SAED pattern with [011] zone axis (bottom-left inset), and an enlarged image of the GB (marked A in the top-right inset). The GB is not smooth but exhibits corrugated configurations with irregular arrangements of small facets, indicative of the high-energy non-equilibrium state [18]. The change of the GB orientation is likely due to operation of multi-slip systems inside the grain with straining. Figure 2(b) is the HREM image from the rectangle part of $(a)$. An amorphous packet is observed to occur along the GB. In particular, the significant distortion of lattice fringes very close to the GB is visible as indicated by an arrowhead. The other portion along the same GB, however, appears ordered and well defined. The ordered GB is very narrow and the width is $\sim 2$ interatomic spaces, i.e. close to the GB width in the conventional coarse-grained materials.

Figure 3(a) gives another example also showing the zigzag GB of an NC grain $\sim 140 \mathrm{~nm}$ in size. Figure $3(b)$ is the lattice image of the rectangle part of $(a)$. It is interesting to note the presence of the localized amorphization along the GB. The amorphous GB is $\sim 30 \mathrm{~nm}$ long but has a width of $\sim 4 \mathrm{~nm}$ perpendicular to the GB. The interfacial chemistry is analysed using the nanoscale energy dispersive $\mathrm{x}$-ray (EDX) 


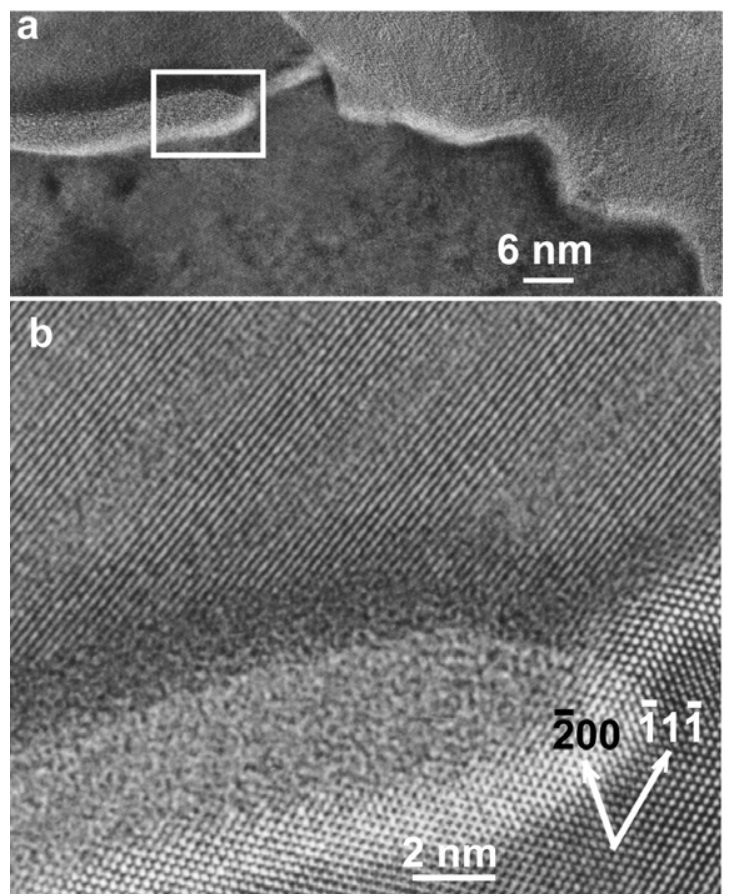

Figure 3. (a) Electron micrograph of a GB. (b) HREM image of the GB of the rectangle part of $(a)$, showing the amorphous phase along the GB.

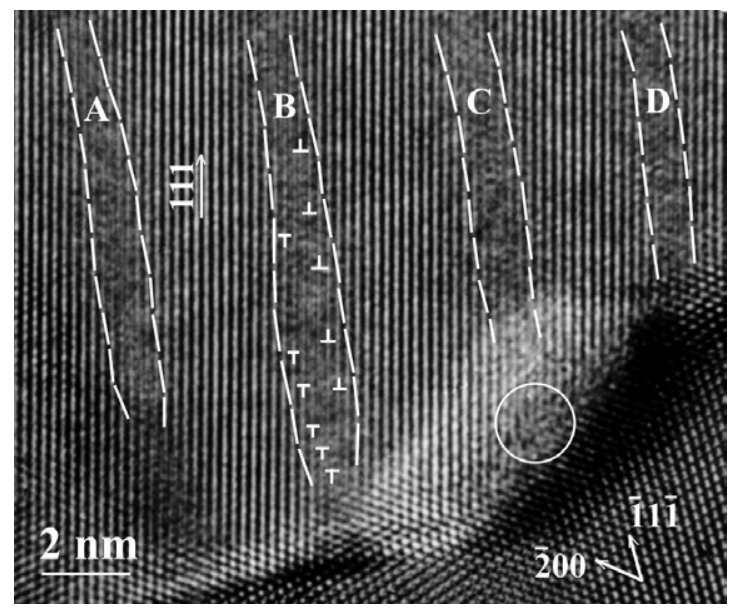

Figure 4. HREM image of the GB with nearby dislocation complexes (marked with dashed lines and labelled A through D). A small disordered area at the GB is circled.

spectroscopy at a $1.2 \mathrm{~nm}$ spatial resolution. The amorphous GB exhibits segregation of $\mathrm{Cu}(6.7 \pm 0.8 \mathrm{wt} \%)$ as compared with the average content of $\mathrm{Cu}(3.6 \pm 0.5 \mathrm{wt} \%)$ inside the grain. Meanwhile, no evidence of melting such as flow features and diffuse boundaries is seen in both figures $2(b)$ and $3(b)$. It is thus concluded that the observed amorphization process occurs as a direct result of the solid-state transformation due to imposed severe deformation.

Since crystal defects play a crucial role in the SSA, the observation of defects may shed new light on the pathway to understand the nature of the SSA. Figure 4 is a lattice image showing a GB. Close inspection reveals that in the vicinity of the GB, there is a group of parallel regions where the lattice

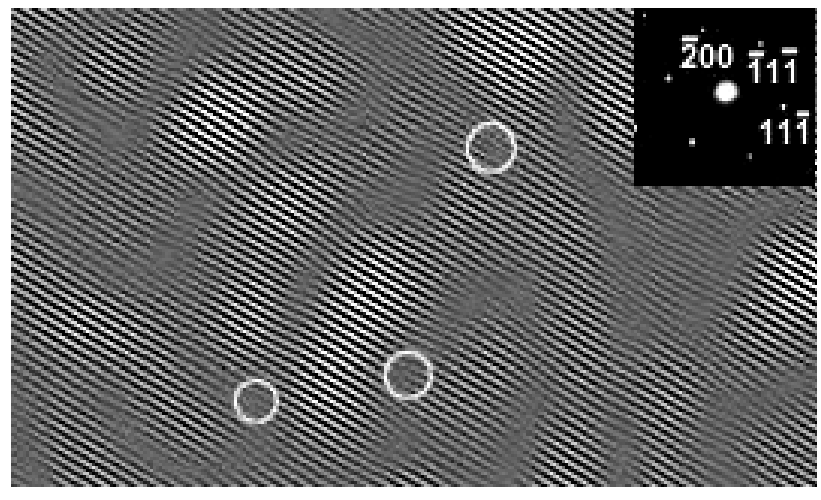

Figure 5. Fourier reconstructed image showing dislocations (a few are circled). Inset is the corresponding SAED pattern with [011] zone axis.

fringes are absent, or at best ill-defined (marked with dashed lines and labelled A through D). Each region is characterized by two-side dislocation (indicated by T) boundaries (e.g. region B) and an intermediate narrow region. The ill-defined nature of the lattice fringes suggests that there may be very high strains in these areas. When a large number of dislocations are present, they tend to form the more complicated configurations, called dislocation complexes, to reduce the elastic energy resulting from their mutual interactions [7]. In particular, the localized lattice disordered area (circled) is exactly visible is at the GB, intersected by a dislocation complex. The adjacent region is, however, a well-defined crystal lattice. It is reasonable to assume that the amorphous phase is more likely to form as a result of the dislocation activity within the dislocation complex. This is supported by the fact that the dislocation complex may cause accumulation of the significantly high density dislocations and highly localized strain during deformation.

Figure 5 is a Fourier reconstructed lattice image taken near the GB of an NC grain $\sim 140 \mathrm{~nm}$ in diameter. One-dimensional [111] fringes are visible. In the inset is the SAED pattern with [011] zone axis. Dislocations (a few are circled) are seen from the extra half-planes of the crystal lattices. The dislocation density is measured to be as high as $11 \times 10^{12} \mathrm{~cm}^{-2}$, using the number of dislocations divided by the area of the figure. The dislocation density on other $(11 \overline{1})$ and $(\overline{2} 00)$ planes are also measured to be $\sim 8.9 \times 10^{12} \mathrm{~cm}^{-2}$ and $\sim 0.48 \times 10^{12} \mathrm{~cm}^{-2}$, respectively. The total dislocation density within this local area is thus around $20 \times 10^{12} \mathrm{~cm}^{-2}$, which is hardly achievable in heavy plastically deformed metals (maximum $10^{12} \mathrm{~cm}^{-2}$ ). Extensive observations indicate that the dislocation density may be as high as the order of magnitude of $30 \times 10^{12} \mathrm{~cm}^{-2}$ in the local region near the GB.

\section{Discussion}

The SMAT process produces heavily strained crystallites on a nanometre scale and high density dislocations are created. The time dependence of the structural changes is currently being undertaken in greater detail. Meanwhile, with the reduction of grain sizes, dislocations are no longer sustained within the grain and, once generated, rapidly diffuse to the GB [19]. Dislocations do not dissociate when they arrive at GBs but 
remain as immobile and disordered arrays of GB dislocations adding to the GB disorder and further increasing the local strain [20]. At higher dislocation density, on the other hand, strong interactions between dislocations lead to the formation of dislocation complexes [7]. The dislocation complexes are energetically favoured as dislocation density increases.

With the accumulation of disorderedly distributed dislocations in the local region of the GB, significantly high elastic strain due to the atomic mismatch will occur at the dislocation core regions. The local lattice distortion may be severe enough where SSA takes place directly. The observed lattice distortion near the amorphous phase in figure 2 strongly suggests that localized SSA probably initiates to release high elastic strain at the dislocation core region. The amorphization is probably a strong relaxation mode of high density dislocations at the GB. Lattice instability due to elastic strain is responsible for the localized SSA. The MD simulation [7] demonstrated that the crystalline defects contribute directly to atomic position disorder and lattice strain. It is their interplay that leads to softening of the elastic modulus and the final collapse of the crystallinity. The presence of many dislocation core regions is possible within the dislocation complex. The intersection of the GB and the dislocation complex will create a localized high strained region that may act as the nucleation site for amorphization. After the amorphous nucleation, continuous deformation during SMAT will induce more dislocations, and those dislocations tend to relax by disordering more lattice sites leading to extending the amorphous phase. Alternatively, one can say that the amorphous phase nucleated from the dislocation core region grows by absorbing more dislocations so as to reduce the energy of the system. The defects provide the thermodynamic driving force for the SSA. The reason for the formation of the partial rather than full amorphization may probably be due to the non-uniform generation of both dislocations and dislocation complexes. Our results, therefore, suggest that localized SSA should be interpreted in terms of defectaccumulation induced disordering, as evident in figures 2 and 4. Similar amorphous nucleation in the dislocation regions was also observed in TiNi after high-pressure deformation [10] and in ball-milled Si [11].

Chemical disordering might play an important role in the amorphous formation during deformation. To clarify this it is necessary to study the change of the degree of long-range order due to deformation, but this was not done in the present paper. It is still not obvious how the defects such as dislocation and GBs are related to solute atoms. The question of whether or not the solute-induced topological defects exist and if they do, how to characterize them remains to be answered. However, the presence of copper will not favour amorphization of aluminium (using the confusion principle enunciated for amorphous formation and so far frequently obeyed), and the amorphization has not been reported in $\mathrm{Al}-\mathrm{Cu}$ systems by either mechanically alloying or ball milling [8]. Besides, the present inspection was focused on $\mathrm{NC}$ grains located at sites at least $\sim 20 \mu \mathrm{m}$ deep below the surface where no foreign impurities are desired during the SMAT.

\section{Conclusions}

In summary, deformation-induced SSA is observed to occur at the GBs in an $\mathrm{NC} \mathrm{Al}$ solid solution. The amorphous phase tends to nucleate from the defective sites. It is believed that lattice instability due to high elastic strain in the dislocation core regions is accounted for by localized SSA. Besides, the $\mathrm{GB}$ of the deformed grains reveals a continuously varying atomic structure. While most of the GB appears ordered, some localized amorphization may occur along the same GB.

\section{Acknowledgments}

This work was supported by the National Natural Science Foundation of China (Grant No 50471086, 10472117, 50021101), National 973 Project of China (Grant No 2004CB619305), The Chinese Academy of Sciences (Grant No KJCX2-SW-L2) and National Center for Nanoscience and Technology, China. Helpful discussions with Professor Ovid'ko are highly acknowledged.

\section{References}

[1] Schwarz R B and Koch C C 1986 Appl. Phys. Lett. 49146

[2] Schultz L 1988 J. Less-Common Met. 145233

[3] Johnson W L 1986 Prog. Mater. Sci. 3081

[4] Bakker H, Zhou G F and Tang H 1995 Prog. Mater. Sci. 39159

[5] Ikeda H, Qi Y, Cagin T, Samwer K, Johnson W L and Goddard W A III 1999 Phys. Rev. Lett. 822900

[6] Ovid'ko I A and Reizis A B 1999 J. Phys. D: Appl. Phys. 32 2833

[7] Li M 2000 Phys. Rev. B 6213979

[8] Suryanarayana C 2001 Prog. Mater. Sci. 461

[9] Novikova E E, Tatyanin Y V and Kurdjumov V G 1995 Scr. Metall. Mater. 33851

[10] Tatyanin E V, Kurdyumov V G 1990 Phys. Status Solidi a 121 455

[11] Huang J Y, Yasuda H and Mori H 1999 Phil. Mag. Lett. 79305

[12] Gutkin M Yu and Ovid'ko I A 1994 Phil. Mag. A 70561

[13] Chen M W, McCauley J W and Hemker K J 2003 Science 299 1563

[14] Lu K and Lu J 1999 J. Mater. Sci. Technol. 15193 Lu K and Lu J 2004 Mater. Sci. Eng. A 375-377 38

[15] Wu X, Tao N, Hong Y, Xu B, Lu J and Lu K 2002 Acta Mater. 502075

[16] Wu X, Tao N, Hong Y, Liu G, Xu B, Lu J and Lu K 2005 Acta Mater. $\mathbf{5 3} 681$

[17] Tao N R, Wang Z B, Tong W P, Lu J and Lu K 2002 Acta Mater. 504603

[18] Horita Z, Smith D J, Furukawa M, Nemoto M, Valiev R Z and Longdon T G 1996 J. Mater. Res. 111880

[19] Rawers J and Cook D 1999 Nanostruct. Mater. 11331

[20] Valiev R Z, Mishral R, Grozal J and Mukherjee A 1996 Scr. Mater. 341443 